\title{
Simultaneous dual-band optical coherence tomography for endoscopic applications
}

\author{
Jianbing Xu, ${ }^{a}$ Luoqin Yu, ${ }^{a}$ Xiaoming Wei, ${ }^{a}$ Xie Wang, ${ }^{a}$ Po Ching Chui, ${ }^{a}$ Kin Tak Chan, ${ }^{b}$ Edmund Y. Lam, ${ }^{c}$ \\ Nikki P. Lee, ${ }^{b}$ and Kenneth K. Y. Wong ${ }^{a, *}$ \\ aUniversity of Hong Kong, Photonic Systems Research Laboratory, Department of Electrical and Electronic Engineering, Pokfulam Road, \\ Hong Kong, China \\ bUniversity of Hong Kong, Department of Surgery, Hong Kong, Pokfulam Road, Hong Kong, China \\ 'University of Hong Kong, Imaging Systems Laboratory, Department of Electrical and Electronic Engineering, Hong Kong, China
}

\begin{abstract}
Dual-band optical coherence tomography (OCT) can greatly enhance the imaging contrast with potential applications in functional (spectroscopic) analysis. A new simultaneous dual-band Fourier domain mode-locked swept laser configuration for dual-band OCT is reported. It was based on a custom-designed dual-channel driver to synchronize two different wavelength bands at 1310 and $1550 \mathrm{~nm}$, respectively. Two lasing wavelengths were swept simultaneously from 1260 to $1364.8 \mathrm{~nm}$ for the 1310-nm band and from 1500 to $1604 \mathrm{~nm}$ for the $1550-\mathrm{nm}$ band at an A-scan rate of $45 \mathrm{kHz}$. Broadband wavelength-division multiplexing was utilized to couple two wavelength bands into a common catheter for circumferential scanning to form dual-band OCT. The proposed dual-band OCT scheme was applied to endoscopic OCT imaging of mouse esophageal wall ex vivo and human fingertip in vivo to justify the feasibility of the proposed imaging technique. The proposed dual-band OCT system is fast and easy to be implemented, which allows for in vivo high-speed biomedical imaging with potential applications in spectroscopic investigations for endoscopic imaging. ๑ 2014 Society of Photo-Optical Instrumentation Engineers (SPIE) [DOI: 10.1117/1.JBO.19.12.126007]
\end{abstract}

Keywords: optical coherence tomography; endomicroscopy; internal organ imaging; Fourier domain mode locked swept laser; spectroscopic analysis.

Paper 140449RR received Jul. 17, 2014; accepted for publication Nov. 10, 2014; published online Dec. 9, 2014.

\section{Introduction}

Optical coherence tomography $(\mathrm{OCT})^{1,2}$ is a noninvasive imaging modality, which allows for cross-sectional structure visualization with high-spatial resolution in the micrometer range. Spectroscopic OCT (SOCT), as a functional extension of OCT, has been introduced to exploit the spectroscopic information of the sample to enhance the image contrast. ${ }^{3-5}$ In addition to the image contrast enhancement, SOCT is also capable of providing additional spectroscopic information, such as the absorption properties of materials ${ }^{6}$ or qualitative scatterer size differentiation in tissues. ${ }^{7}$

The conventional methods of SOCT primarily extract the spectroscopic information within one wavelength band only., Compared with a single wavelength band scheme, a dual-band scheme is advantageous in that it offers higher wavelength differences by simultaneously imaging at two distinct wavelength regions and potentially enables higher imaging contrast. Moreover, it allows easy implementation of real-time image processing. ${ }^{8}$ The key enabling part of the dual-band scheme is the dual-band laser source. Simultaneously, OCT imaging at two distinct wavelength bands have been reported in timedomain, ${ }^{8}$ full-field, ${ }^{9}$ spectral-domain,,${ }^{10,11}$ and polygon-based swept source. ${ }^{12}$ However, the previously reported dual-band OCT systems may not be suitable for the endoscopic applications either due to the short imaging range, speed limitation, or computational complexity. Extending the current dualband OCT scheme to the endoscopic applications is strongly

*Address all correspondence to: Kenneth K. Y. Wong, E-mail: kywong@eee .hku.hk desirable. First, for intravascular applications where the signal collection efficiency is not as good as the galvanometer-based scanner system, the imaging contrast can be improved by employing a dual-band scheme. Second, a relatively long imaging range is required for the endoscopic applications due to the various lumen sizes of the tissue, and high sensitivity and speed are generally required to obtain an acceptable image quality. However, previously reported dual-band OCT was deployed in the spectral domain, and therefore, has limited imaging range, speed and sensitivity. For endoscopic applications such as when blood vessels are imaged, a rapid injection of angiographic contrast fluid is used to temporarily displace the blood and provide a clear imaging field, and it is more suitable to use swept-source OCT (SS-OCT) system due to its higher speed $^{13}$ and relatively larger imaging range $(\sim 2 \mathrm{~cm}) .{ }^{14} \mathrm{~A}$ dual-band SS-OCT system based on fiber optical parametric amplifier (OPA) has been reported by our group. ${ }^{15}$ However, it was predominately limited by the available gain spectrum of fiber-based OPA, and therefore, limited the resolution of the dual-band SS-OCT system.

These limitations can be overcome by a dual-band laser source based on Fourier domain mode locked (FDML) laser, ${ }^{16}$ which has been reported to overcome the physical limitations of traditional swept laser sources in regard to the speed and imaging range. Therefore, it is worthwhile to implement a dual-band FDML laser source for endoscopic applications.

Here, we present a simultaneous dual-band laser source based on FDML swept laser for endoscopic OCT. The two wavelength bands are illuminated simultaneously on the sample

0091-3286/2014/\$25.00 (C) 2014 SPIE 
and the spectral responses of the sample are recorded and colorencoded for improved image contrast. Proof-of-concept imaging experiments based on our initial design in a galvanometerscanned system have been reported before. ${ }^{17}$ Here, ex vivo OCT imaging of mouse esophagus using the proposed dualband OCT system is further investigated to justify the feasibility of the proposed imaging technique in the endoscopic scenario. The proposed dual-band OCT system is fast and easy to implement and allows for in vivo high-speed biomedical imaging with potential application in spectroscopic investigations.

\section{Methods}

The overall system schematic of the proposed dual-band FDML swept laser source is shown in Fig. 1. It consisted of two semiconductor optical amplifiers (SOA, Inphenix) as the gain medium inside the cavity. A polarization controller was inserted into the cavity to control the state-of-polarization. Two fiber Fabry-Perot tunable filters (FFP-TF by Micron Optics) centered at 1310 and $1550 \mathrm{~nm}$, respectively, were incorporated as a narrow band filter for active wavelength selection. They have a free spectral range (FSR) of $200 \mathrm{~nm}$ and a finesse of 1000 and were tuned periodically at the cavity round-trip time, respectively. Two isolators were inserted, respectively, to ensure unidirectional lasing in each cavity. Fifty percent of the dual-band FDML laser source was coupled out via the corresponding output coupler and amplified by two booster optical amplifiers (BOA, COVEGA) before they were combined together to launch to the OCT system. The fiber delay line (SMF28e, Corning) was $4.5-\mathrm{km}$ long which corresponds to a swept rate of $\sim 45 \mathrm{kHz}$. A trigger delay can be adjusted to exactly cancel the forward sweep of the bidirectional FDML swept laser source, which will be discussed further in the subsequent paragraphs.

A custom-designed dual-channel driver generates two sinusoidal signals, of which amplitude and offset were individually tuned to optimize the laser performance, while the frequency was set to be the same to ensure the synchronization between the two FFP-TFs. The fiber delay line of $4.5 \mathrm{~km}$ was commonly shared by two laser cavities via two broadband 1310/1550

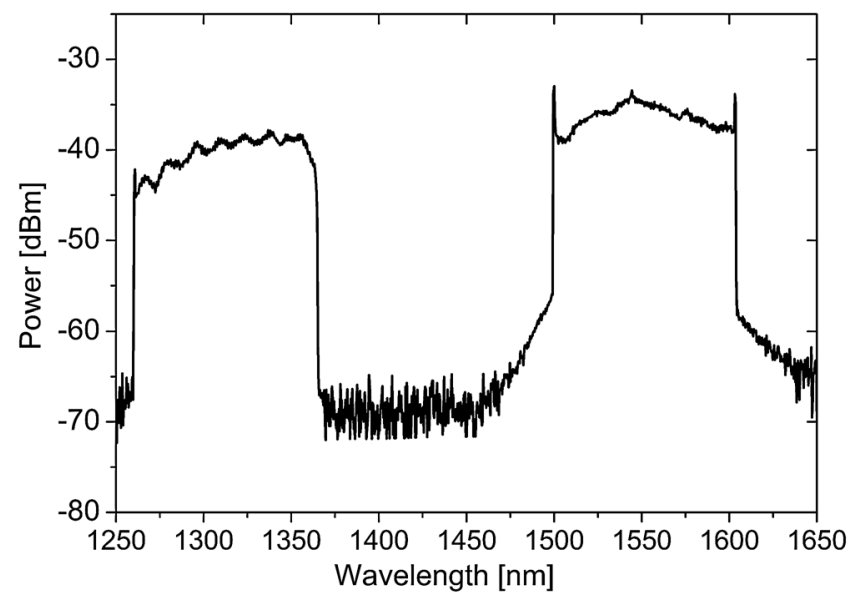

Fig. 2 Measured spectrum of the dual-band FDML swept laser.

wavelength-division multiplexing (WDM) couplers. It should be noted that the total cavity length of the two laser cavities has to be maintained to be equivalent such that the optimized swept frequency is the same.

The integrated output spectrum of the dual-band FDML swept laser before the BOA was measured by an optical spectrum analyzer in the peak-hold mode is shown in Fig. 2. For the $1310-\mathrm{nm}$ band, the spectrum spanned a range from 1260 to $1364.8 \mathrm{~nm}$, approximately $105 \mathrm{~nm}$. The full-width at half-maximum (FWHM) was measured to be $\sim 74 \mathrm{~nm}$. For the $1550-\mathrm{nm}$ band, the spectrum spanned a range from 1500 to $1604 \mathrm{~nm}$, $\sim 104 \mathrm{~nm}$. The FWHM was measured to be $\sim 70 \mathrm{~nm}$.

Measured average output powers of 50.5 and $21.6 \mathrm{~mW}$ were obtained for the 1310- and 1550-nm bands after the BOA, respectively. Signal-to-noise ratios of $44.2 \mathrm{~dB}$ for $1310 \mathrm{~nm}$ and $41.7 \mathrm{~dB}$ for $1550 \mathrm{~nm}$ were obtained when the optical powers illuminating on the sample were $\sim 14$ and $6 \mathrm{~mW}$, respectively. Both bands have sensitivity of $\sim 92 \mathrm{~dB}$. The measured sensitivity is about $13 \mathrm{~dB}$ lower than the theoretical value calculated at shot noise limited $(\sim 105 \mathrm{~dB})$ detection ${ }^{13}$ since the thermal noise

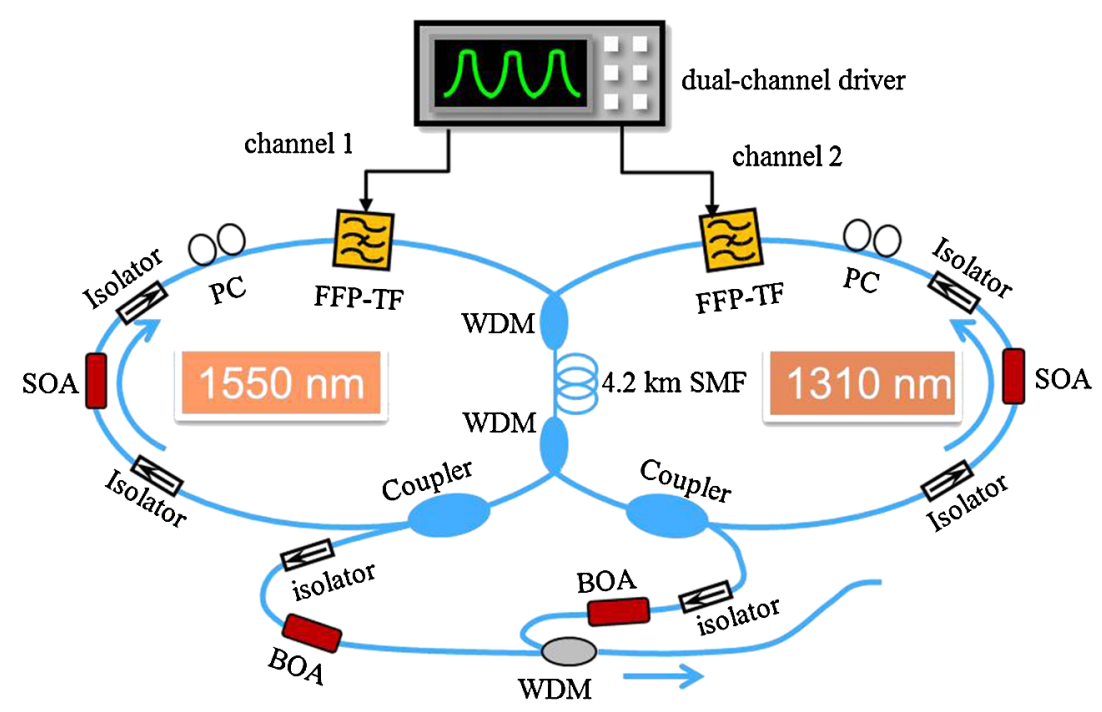

Fig. 1 Schematic diagram of the dual-band Fourier domain mode-locked (FDML) swept laser ring cavity. SOA, semiconductor optical amplifier; FFP-TF, fiber Fabry-Perot tunable filter; SMF, single-mode fiber; ISO, isolator; PC, polarization controller; BOA, booster optical amplifier; WDM, wavelength-division multiplexing. Blue line refers to fiber transmission. Black line represents electronic-based transmission. 
is not significant and excess photon noise can be cancelled out in the balance-detected OCT system. The discrepancy can be partially explained by the insertion loss of the setup, including free-space coupling loss, fiber component loss [Broadband 1310/1550 WDM couplers, custom-made probe interface unit (PIU)], and noise introduced by the data acquisition board and detector. For clinical imaging, the requirements for sensitivity may even be higher than in a laboratory setting, and further optimization of the system loss and detection efficiency is essential to further increase the sensitivity of the system. The measured axial resolutions for the 1310- and 1550-nm bands were $\sim 12$ and $\sim 18 \mu \mathrm{m}$, respectively. We have also measured the roll-off curve for both directions of both wavelength bands. For both directions, the roll-off curve shows a slower decay for the backward direction. Therefore, we use the backward direction of both wavelength bands for the imaging. The 6-dB sensitivity drop points (half the coherence length) for the 1310 and $1550 \mathrm{~nm}$ band are 4.3 and $3.8 \mathrm{~mm}$, respectively. For intravascular OCT applications where a large coherence length is required for optimal imaging of large blood vessels, further improvement of the coherence length of the proposed dual-band swept laser can be expected by incorporating dispersion management inside the cavity. ${ }^{18}$

Figure 3 shows the experimental setup of the 1310/1550 dual-band FDML swept laser source for dual-band OCT. The dual-band FDML swept laser was connected to two optical circulators and the output was then connected to a broadband $1310 / 1550$ WDM coupler. It should be pointed out that $5 \%$ of the dual-band FDML swept laser was split off and coupled into two separated Mach-Zehnder interferometers with an FSR of $103 \mathrm{GHz}$ for recalibration of time to optical frequency. A balanced detector was employed to suppress the background noise and to double the amplitude. The signals were acquired on a high-speed digital acquisition card (ATS460, Alazartech, Montreal) with a 14-bit resolution and sampling speed up to $125 \mathrm{MS} / \mathrm{s}$. The resampling and fast Fourier transform (FFT) procedures were performed by a graphical processing unit card (GPU, GTX460, NVIDIA) inserted into a standard personal computer (dual core, Dell) as described in our previous demonstration. ${ }^{15,19}$ The spline interpolation and FFT algorithms were programmed in the CUDA 2.3 environment and packaged into a dynamic link library for calling by LabVIEW codes. The processing speed under the LabVIEW environment was sufficient to perform real-time ex vivo imaging in our setup.
Especially noteworthy is the fact that we used two BDs for simultaneous detection ${ }^{12}$ instead of the time-multiplexing scheme which was reported in our previous work ${ }^{15}$ as it will inevitably lose some power due to the extra fiber delay line, ${ }^{15}$ and therefore, degrade the sensitivity of the system.

A custom-made PIU containing a fiber-optic rotary joint and a fiber-optic catheter (Dragonfly, Light lab Imaging) was used to interface with the dual-band FDML laser source. Therefore, real-time synchronized circumferential scanning can be achieved for the proposed dual-band OCT scheme.

\section{Results and Discussions}

To validate the feasibility of the proposed dual-band OCT scheme, we obtained ex vivo images on the esophageal wall of mouse. The catheter was inserted into the esophagus for circumferential imaging. Simultaneous imaging was achieved by circumferentially scanning the beam with the custom-designed PIU. The imaging speed was 90,000 A-scans/s with each circumferential frame consisting of $2000 \mathrm{~A}$-scans and each A-scan of 2048 pixels. As a result, the circumferential frame rate was 45 frames/s.

Figures 4(a) and 4(b) were obtained at 1310- and 1550-nm wavelength bands, respectively. Two-dimensional (2-D) circumferential ex vivo OCT images of the mouse esophagus permit excellent differentiation of different layers of esophageal wall. The mucosa was readily visualized owing to its low optical backscattering compared with the submucosa (SM). It is critical to differentiate mucosa versus SM, especially in determining tumor stage in gastric and esophageal cancers. ${ }^{20}$ The stratum corneum (SC) contains a high keratin content and provides strong scattering, while the epithelium (EP) appeared as a low scattering layer in the OCT image. The muscularis mucosa (MM) layer between the lamina propria (LP) and SM is a less reflective layer as it predominantly comprises skeletal muscle, while LP and SM exhibit strong scattering and appear as a bright layer since they contain abundant collagen fibers. ${ }^{21,22}$ The penetration depth of the 1550-nm band is slightly larger than that of the 1310-nm band, and the axial resolution of the 1310-nm band is obviously better than that of the $1550-\mathrm{nm}$ band, which correlates well with the theoretical prediction. In consequence of the better resolution of the 1310-nm wavelength band, the visibility of LP is better in Fig. 4(a). Furthermore, MP is more visible in the deeper regions of the esophagus.

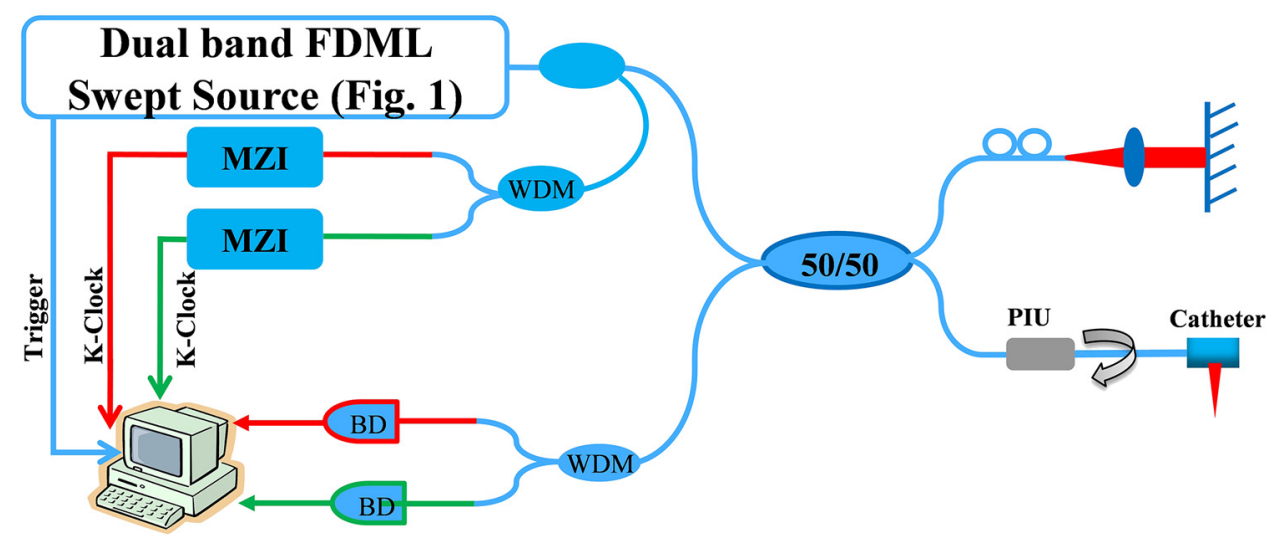

Fig. 3 Schematic diagram of the dual-band spectroscopic OCT system. BD, balanced detector; MZI, Mach-Zehnder interferometer; WDM, wavelength-division multiplexer; PIU, probe interface unit. Red lines refer to $1310-\mathrm{nm}$ band while green refers to $1550-\mathrm{nm}$ band. 

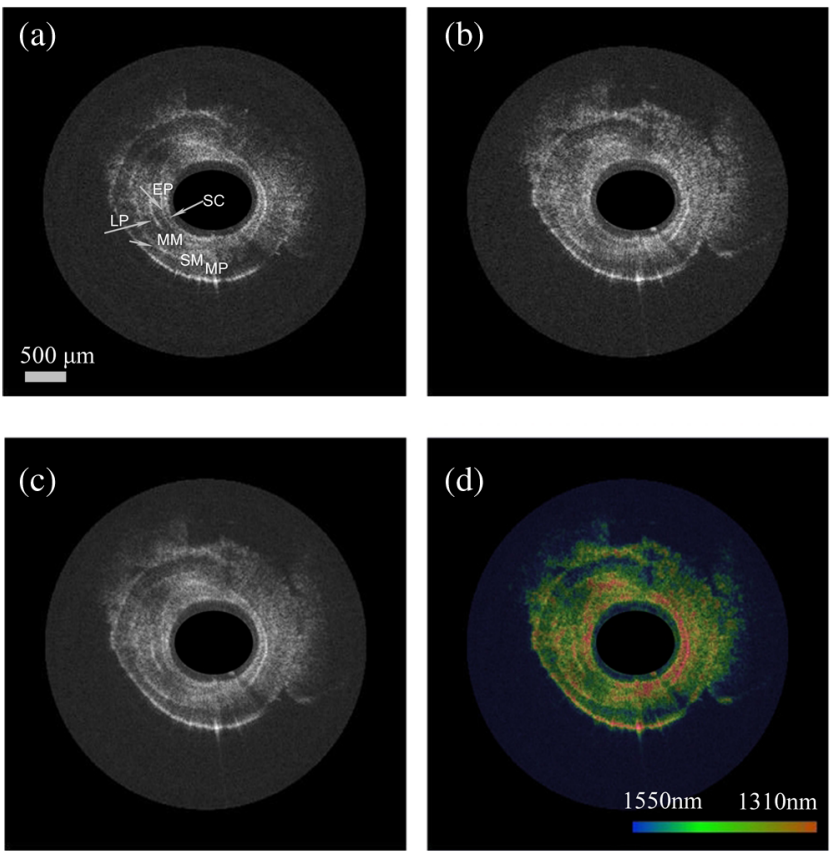

Fig. 4 Ex vivo images of mouse esophageal wall at $1310 \mathrm{~nm}$ (a) and $1550 \mathrm{~nm}$ (b) band, (c) frequency compounded images of both wavelength bands, and (d) differential color-encoded image. SC, stratum corneum; EP, epithelium; LP, lamina propria; MM, muscularis mucosa; SM, submucosa; MP, muscularis propria.

The image in Fig. 4(c) was frequency-compounded by calculating the pixel-wise average of the intensity values of both wavelength bands. One can observe that the speckle noise was also reduced owing to the frequency compounding of the uncorrelated speckle pattern of different wavelength bands. ${ }^{23}$ It should be pointed out that the OCT images in Figs. 4(a) and 4(b) had to be scaled in depth to be congruent to each other as the scanning depth ranges of two images were different. An estimated average refractive index of 1.4 for the tissue for both bands is used. Further co-registration of the dual band images is done by carefully choosing the scale factor, which is determined by the different axial resolutions/coherence lengths of the two wavelength bands. ${ }^{24}$

The color-encoded image, as shown in Fig. 4(d), combines the advantage of two different wavelength bands, which is the relatively deeper penetration depth of the 1550-nm band and higher axial resolution of the $1310 \mathrm{~nm}$ band, and enhances the image contrast in comparison with the respective image by only one wavelength band. To obtain the color-encoded OCT image, the average intensity of the two images was set to be a $V$ value in a hue value saturation map. ${ }^{24}$ Color information was generated by coding the intensity difference of the two bands to the hue value $H$. Note that both images were Wiener filtered before the intensity difference was calculated to minimize the effect of speckle noise on the color interpretation. The saturation $S$ ranged from 0 to 1 . It was set to be a constant value of 0.5 here. $^{24}$

To visualize the backscattering in both spectral regions, a color-encoded differential image is calculated as shown in Fig. 4(d). The intensity difference of the two bands is coded as a different color value. The additional image contrast of the proposed dual-band method coincides with different tissue layers. The additional color contrast observed in the esophageal wall ex vivo image accentuates the distinction between the LP and SM layers. This region is especially relevant for diagnostics of normal and abnormal mucosa in the esophagus. Thus, the simultaneous dual-band OCT approach can have a significant impact for identifying and distinguishing malfunctioned parts. The tissue surface appears as red layers, discriminating the SC from the EP layers below. By contrast, the outer region is colored green, indicating higher penetration for the $1550 \mathrm{~nm}$ wavelength region. The mucosa alternates from red to green, indicating different layers. Thus, the mucosal layer morphology can be easily identified by using spectroscopic information. The difference could be caused by the local water amount, collagen, and muscles. An enhanced penetration depth at $1550 \mathrm{~nm}$ due to less tissue scattering is clearly observable, revealing the MP layer that is not visible at $1310 \mathrm{~nm}$. On the other hand, the OCT image for $1310 \mathrm{~nm}$ shows a higher resolution due to the shorter wavelength. The thin LP layer between the EP and the MM appears sharper in this spectral region.

The proposed dual-band FDML laser source for esophagus endoscopy is promising for least in two aspects. First, the earlystage cancer usually involves some changes of the mucosal layer as reflected by tissue optical properties (such as absorption or scattering), thus it can be probed by the dual-band SOCT by quantifying absorption or scattering. Second, as the conventional OCT image contrast is low, contrast enhancement via dual-band OCT is capable of improving the diagnostic capability of a conventional single-band OCT. This additional contrast can be generated with only a few decibels of intensity difference between the two spectral bands. In contrast to our previous work, ${ }^{15}$ the increased separation in the central wavelength of the two spectral bands allows for an enhanced image contrast. In comparison with the current reported literatures about a dual-band spectral domain OCT system, the acquisition time is shorter and the imaging range is reasonably longer owing to the utility of the FDML swept laser, enabling potential in vivo diagnosis with low computational costs in intravascular applications where a long imaging range is required. ${ }^{25}$ Currently, our system has limited capabilities in terms of spectroscopic contrast due to the limited absorption difference at these two distinct wavelength bands. The drawback can be overcome by using spectral bands with a larger spectroscopic contrast, such as 1550/1060 nm. ${ }^{15}$ Compared with the reported high speed dual-band OCT work, ${ }^{26}$ the proposed setup has a limited speed due to the achievable driving frequency when a large sweep amplitude has to be applied to the FFP-TF to achieve a large sweeping bandwidth. Further speed enhancement of the FDML swept laser can be achieved by adopting the external-buffering technique. ${ }^{27}$

As a second example, simultaneous dual-band OCT imaging of the human fingertip in vivo is presented in Fig. 5. The images were obtained by pressing two fingertips against the imaging catheter tip. It is evident that the spatial resolution achieved at $1310-\mathrm{nm}$ band is higher than that at the 1550-nm band, as expected. On the other hand, there is an obvious benefit in penetration depth at the 1550-nm band. The same abovementioned color encoding method for Fig. 4 was applied. Because the spectral features of the sample appeared different for different wavelength bands, the combined dual-band image will enhance the imaging contrast due to its spectral characteristics. As shown in the figure, the epidermal and dermal regions can be easily separated with an enhanced contrast. 

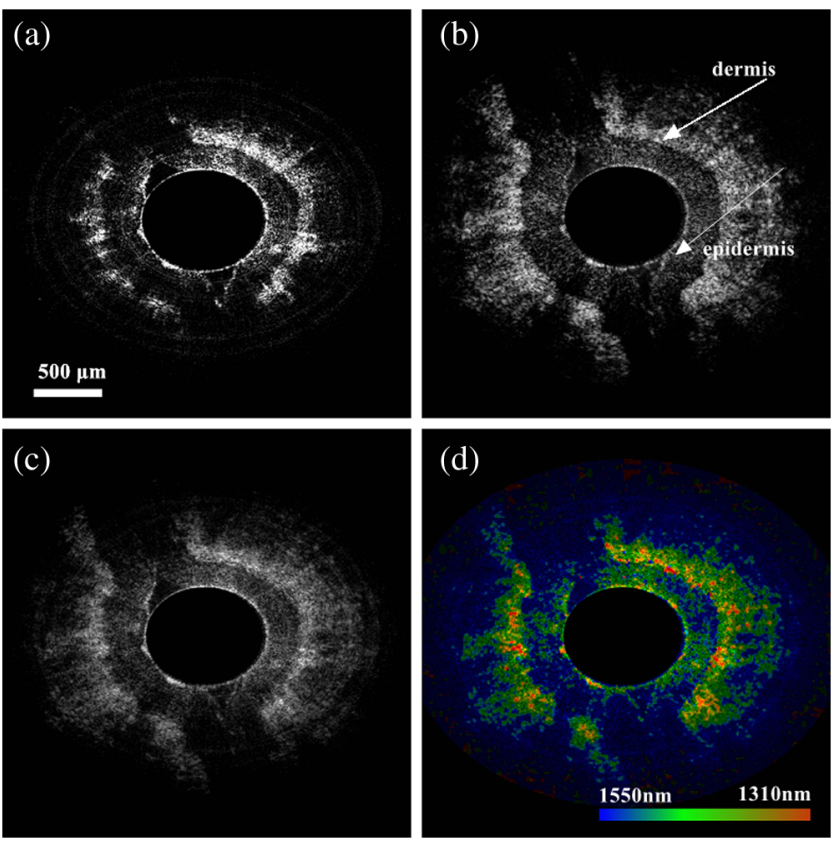

Fig. 5 In vivo images of human fingertip at $1310 \mathrm{~nm}$ (a) and $1550 \mathrm{~nm}$ (b) band, (c) frequency compounded images of both wavelength bands, and (d) differential color-encoded image.

\section{Conclusion}

In summary, we have proposed and developed a simultaneous dual-band OCT imaging system based on Fourier domain mode locked laser for endoscopic applications for the first time. Using a fiber probe, the method allows improved imaging contrast with low computational costs. We have demonstrated imaging of mouse esophagus and human fingertip using the proposed dual-band FDML swept laser sources and presented preliminary observations. The principle can be extended to other wavelengths as well and can be expected to be used in other measurements, such as tissue oxygenation, ${ }^{28}$ and clinical applications such as decisive tumor diagnosis. Future work includes evaluation of the imaging scheme for the detection of diseases, such as dysplasia in Barrett esophagus.

\section{Acknowledgments}

This research was partially supported by grants from Research Grants Council of the Hong Kong Special Administrative Region, China (Project No. HKU 7172/12E) and ITF Tier 3 Project (ITS/189/13). The authors would like to acknowledge Peter Cimalla from University of Technology Dresden, Germany, for the helpful discussions. We would also like to acknowledge the help of Rui Zhu from Shenzhen Vivolight Medical Device, Ltd., China for providing the custom-designed PIU.

\section{References}

1. D. Huang et al., "Optical coherence tomography," Science 254(5035), 1178 (1991).

2. A. Unterhuber et al., "Simultaneous dual wavelength eye-tracked ultrahigh resolution retinal and choroidal optical coherence tomography," Opt. Lett. 38(21), 4312-4315 (2013).

3. U. Morgner et al., "Spectroscopic optical coherence tomography," Opt. Lett. 25(2), 111-113 (2000).
4. A. L. Oldenburg, C. Y. Xu, and S. A. Boppart, "Spectroscopic optical coherence tomography and microscopy," IEEE J. Sel. Top. Quant. Electron. 13(6), 1629-1640 (2007).

5. F. E. Robles et al., "Molecular imaging true-colour spectroscopic optical coherence tomography," Nat. Photonics 5(12), 744-747 (2011).

6. R. Leitgeb et al., "Spectral measurement of absorption by spectroscopic frequency-domain optical coherence tomography," Opt. Lett. 25(11), $820-822$ (2000).

7. B. C. M. Tay et al., "Dual-window dual-bandwidth spectroscopic optical coherence tomography metric for qualitative scatterer size differentiation in tissues," IEEE Trans. Biomed. Eng. 59(9), 2439-2448 (2012).

8. F. Spöler et al., "Simultaneous dual-band ultra-high resolution optical coherence tomography," Opt. Express 15(17), 10832-10841 (2007).

9. D. Sacchet et al., "Simultaneous dual-band ultra-high resolution full-field optical coherence tomography," Opt. Express 16(24), 1943419446 (2008).

10. S. Kray et al., "High-resolution simultaneous dual-band spectral domain optical coherence tomography," Opt. Lett. 34(13), 1970-1972 (2009).

11. P. Cimalla et al., "Simultaneous dual-band optical coherence tomography in the spectral domain for high resolution in vivo imaging," Opt. Express 17(22), 19486-19500 (2009).

12. Y. X. Mao et al., "Simultaneous dual-wavelength-band common-path swept-source optical coherence tomography with single polygon mirror scanner," Opt. Lett. 36(11), 1990-1992 (2011).

13. T. Klein et al., "Multi-MHz retinal OCT," Biomed. Opt. Express 4(10), 1890-1908 (2013).

14. D. C. Adler et al., "Extended coherence length Fourier domain mode locked lasers at $1310 \mathrm{~nm}$," Opt. Express 19(21), 20930-20939 (2011).

15. R. Zhu et al., "Dual-band time-multiplexing swept-source OCT based on optical parametric amplification," IEEE J. Sel. Top. Quant. Electron. 18(4), 1287-1292 (2012).

16. R. Huber, M. Wojtkowski, and J. G. Fujimoto, "Fourier domain mode locking (FDML): a new laser operating regime and applications for optical coherence tomography," Opt. Express 14, 3225-3237 (2006).

17. R. Zhu et al., "Dual-band FDML laser for swept source spectroscopic OCT," in Biomedical Optics, OSA Technical Digest, Miami, Florida, paper BW2A.2 (2012).

18. D. C. Adler et al., "Extended coherence length Fourier domain mode locked lasers at $1310 \mathrm{~nm}, "$ Opt. Express 19(21), 20930-20939 (2011).

19. J. Xu et al., "In vivo OCT imaging based on La-codoped bismuth-based erbium-doped fiber," IEEE Photonics Tech. Lett. 25(17), 1741-1743 (2013).

20. J. G. Fujimoto et al., "Optical coherence tomography: an emerging technology for biomedical imaging and optical biopsy," Neoplasia 2(1), 9-25 (2000).

21. J. Xi et al., "Diffractive catheter for ultrahigh-resolution spectral domain volumetric OCT imaging," Opt. Lett. 39, 2016-2019 (2014).

22. A. Alex et al., "Characterization of eosinophilic esophagitis murine models using optical coherence tomography," Biomed. Opt. Express 5(2), 609-620 (2014).

23. E. Pircher et al., "Speckle reduction in optical coherence tomography by frequency compounding," J. Biomed. Opt. 8(3), 565-569 (2003).

24. P. Cimalla et al., "Simultaneous dual-band optical coherence tomography in the spectral domain for high resolution in vivo imaging," Opt. Express 17(22), 19486-19500 (2009).

25. T. Wang et al., "Intravascular optical coherence tomography imaging at 3200 frames per second," Opt. Lett. 38(10), 1715-1717 (2013).

26. T. S. Kim et al., "Dual-wavelength band spectroscopic optical frequency domain imaging using plasmon-resonant scattering in metallic nanoparticles," Opt. Lett. 39(10), 3082-3085 (2014).

27. R. Huber, D. C. Adler, and J. G. Fujimoto, "Buffered Fourier domain mode locking: unidirectional swept laser sources for optical coherence tomography imaging at 370,000 lines/s," Opt. Lett. 31(20), 2975-2977 (2006).

28. X. Liu and J. U. Kang, "Depth resolved blood oxygen saturation assessment using spectroscopic common-path fourier domain optical coherence tomography," IEEE Trans. Biomed. Eng. 57(10), 2572-2575 (2010).

Jianbing $\mathrm{Xu}$ received his BS degree in electronics from Huazhong University of Science and Technology. He received his PhD degree at the Department of Electrical and Electronic Engineering at the 
University of Hong Kong. His research is mainly focused on optical coherence tomography from system design to spectroscopic applications.

Luoqin Yu received her BS degree in optical science and engineering from Fudan University, Shanghai, China, in 2013. She is currently working toward the MPhil degree in the Department of Electrical and Electronic Engineering, University of Hong Kong, Pokfulam, Hong Kong. Her research interests include Fourier domain modelocked laser, optical time-stretch, and optical coherence tomography.

Edmund $\mathrm{Y}$. Lam received the $\mathrm{BS}, \mathrm{MS}$, and $\mathrm{PhD}$ degrees in electrical engineering from Stanford University, and is now a professor in electrical and electronic engineering at the University of Hong Kong. He has broad research interests around the theme of computational optics and imaging, particularly its applications in semiconductor manufacturing and biomedical systems. He is a fellow of SPIE and OSA.

Kenneth K. Y. Wong received combined BE degree in EE and BS degree in physics from University of Queensland, Australia. $\mathrm{He}$ received the MS and $\mathrm{PhD}$ degrees both in EE at Stanford University and is now an associate professor in electrical and electronic engineering at the University of Hong Kong. He is author or coauthor of over 250 journal and conference papers. He is a senior member of the OSA, IEEE, and IEEE Photonics Society.

Biographies of the other authors are not available. 